\title{
ALICE: a randomized placebo-controlled phase II study evaluating atezolizumab combined with immunogenic chemotherapy in patients with metastatic triple-negative breast cancer
}

\author{
J. A. Kyte ${ }^{1,2^{*}} \mathbb{D}$, A. Røssevold ${ }^{3}$, R. S. Falk ${ }^{4}$ and B. Naume $e^{3,5}$
}

\begin{abstract}
Background: Immunotherapy with checkpoint inhibitors (CI) represents an important novel development in cancer treatment. Metastatic triple-negative breast cancer (mTNBC) is incurable, with a median survival of only 13 months. We have initiated the randomized placebo-controlled phase Ilb study ALICE, evaluating PD-L1 blockade combined with immunogenic chemotherapy in mTNBC patients $(n=75)$. Intriguingly, the host immune response is strongly predictive for the effect of chemotherapy in $\mathrm{MTNBC}$. In the ALICE trial, we release the brake on the immune response by use of atezolizumab, an inhibitory antibody against PD-L1. We utilize anthracyclines, shown to trigger the immune system, and low-dose cyclophosphamide, which has been reported to counter immunosuppressive cells.

Methods: ALICE is a randomized, double-blind, placebo-controlled exploratory phase II study evaluating the safety and efficacy of atezolizumab when combined with immunogenic chemotherapy in subjects with mTNBC. The trial will enroll 75 evaluable subjects, randomized 2:3 into two arms (A:B). The patients receive identical chemotherapy, i.e. pegylated liposomal doxorubicin (PLD $20 \mathrm{mg} / \mathrm{m}^{2}$ intravenously every 2nd week) + cyclophosphamide (50 mg per day, first 2 weeks in each 4 week cycle). Patients in arm A receive placebo, while patients in arm B receive atezolizumab. The primary objectives are assessment of toxicity and progression-free survival. The secondary objectives include overall survival, tumor response rate, clinical benefit rate, patient reported outcomes, biomarkers and assessment of tumor-immune evolution during therapy.

Discussion: The question of how $\mathrm{Cl}$ should be combined with chemotherapy, is a key challenge facing the field. There is a strong preclinical rationale for exploring if anthracyclines, which are considered to induce immunogenic cell death, synergize with PD-L1 blockade, and if low-dose cyclophosphamide counters tumor tolerance. However, the data from patients is as yet very limited, and the clinical evaluation of these hypotheses is among the key objectives in the ALICE trial. The study includes extensive biobanking and translational sub-projects, also addressing other clinically important questions. These analyses may uncover mechanisms of drug efficacy or tumor resistance, and identify biomarkers allowing personalized therapy. If the trial suggests acceptable safety of the ALICE therapy and provide a signal of clinical efficacy, further studies are warranted.
\end{abstract}

*Correspondence: jonky@ous-hf.no

${ }^{1}$ Department of Clinical Cancer Research, Oslo University Hospital, Oslo, Norway

Full list of author information is available at the end of the article

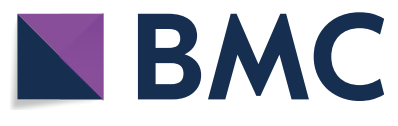

(c) The Author(s) 2020. This article is licensed under a Creative Commons Attribution 4.0 International License, which permits use, sharing, adaptation, distribution and reproduction in any medium or format, as long as you give appropriate credit to the original author(s) and the source, provide a link to the Creative Commons licence, and indicate if changes were made. The images or other third party material in this article are included in the article's Creative Commons licence, unless indicated otherwise in a credit line to the material. If material is not included in the article's Creative Commons licence and your intended use is not permitted by statutory regulation or exceeds the permitted use, you will need to obtain permission directly from the copyright holder. To view a copy of this licence, visit http://creativeco mmons.org/licenses/by/4.0/. The Creative Commons Public Domain Dedication waiver (http://creativecommons.org/publicdomain/ zero/1.0/) applies to the data made available in this article, unless otherwise stated in a credit line to the data. 
Trial registration NCT03164993, May 24th 2017; https://clinicaltrials.gov/ct2/show/record/NCT03164993

Keywords: Breast cancer, Triple negative, Immunotherapy, Checkpoint inhibitor, Immunogenic cell death, PD-1, PDL1, Anthracycline, Cyclophosphamide

\section{Background}

The therapeutic options for metastatic triple-negative breast cancer (TNBC) are very limited. Interestingly, the host immune response is strongly predictive for the effect of chemotherapy in patients with TNBC [1]. In the present trial, we aim at releasing the brake on the immune response by the use of atezolizumab, an inhibitory antibody against Programmed Death Ligand-1 (PD-L1).

Immunotherapy with checkpoint inhibitors produces clinically important responses in several cancer forms, amid limited adverse effects [2-4]. This includes durable responses and improved survival in metastatic cancers. In 2019, immunotherapy was for the first time approved for use against breast cancer (BC), as atezolizumab was approved by the FDA and EMA for use in metastatic TNBC patients, based on the IMPASSION130 trial combining atezolizumab with nabpaclitaxel $[5,6]$. This was the first randomized study demonstrating efficacy of a immunotherapy against TNBC. It should still be noted, that an effect was only found in the patients with PD-L1 expression up front, as measured by the SP142-assay. In the ALICE trial, we aim at triggering sensitivity to atezolizumab in patients that are otherwise not responsive, by use of selected chemotherapy, hypothesized to induce immunogenic cell death and counter immuno-suppressive cells.

There is compelling evidence from animal studies, supported by data from humans, that some chemotherapeutic agents are immunogenic [7-11]. Doxorubicin and cyclophosphamide have been suggested to be particularly powerful inducers of immunogenic cell death. Both agents fulfill $5 / 5$ criteria established for assessing the immunogenicity of different chemotherapeutic drugs (Table 1 in [9]). There is also evidence from humans, particularly in breast cancer, indicating that the clinical effect of doxorubicin and cyclophosphamide depends on the host immune response [11]. Further, these agents have been shown to induce a Type I interferon immune response in breast cancer $[8,10]$. In the present trial, we apply a low-dose, metronomic cyclophosphamide regime, that has been reported to counter immune suppression mediated by regulatory $\mathrm{T}$ cells (Tregs) and myeloid derived suppressor cells (MDSCs) [12]. Taken together, there is a strong rationale for synergy between the applied doxorubicin/cyclophosphamide regime and PD-L1 blockade [7].
PD-L1 is upregulated by IFN $\gamma$-related pathways, and the expression of PD-L1 is usually associated with immune activation. The fact that IMPASSION130 did not show an effect against PD-L1 negative TNBC, highlights the need to explore if more immunogenic chemotherapy, as employed in ALICE, can make immunologically "cold" tumours responsive to PD1/PDL1-blockade. Results from recent TNBC trials suggest that anthracyclines may be well suited for this purpose. The TONIC trial compared different regimes of induction therapy before PD1-blockade. Here, the group receiving doxorubicin recorded the highest response rate to nivolumab (antiPD1), compared to other chemotherapy, radiation or no induction therapy [13]. There was, moreover, evidence of immune activation in the tumour after doxorubicin treatment. The Keynote 522 trial, which was conducted in the neoadjuvant setting, showed significantly increased response rates for the group receiving aPD1 as add-on to chemotherapy [14]. By contrast, the NEOTRIP trial did not show any benefit of anti PD-L1 (Gianni et al. SABCS December 2019). Interestingly. the chemotherapy regime in Keynote 522 contained anthracyclines and cyclophosphamide, while the NEOTRIP chemotherapy consisted only of taxanes. There are several possible explanations to these observations, and no conclusion can yet be drawn on which chemotherapy to combine with PD1-blokade. However, these clinical trial data, as well as the preclinical data mentioned above, suggests that the strategy of combining antracyclins with PD-L1 blockade is worth investigating, also in PD-L1 negative tumours.

\section{Methods \\ Objectives}

Primary objectives:

1. Assessment of toxicity of combined treatment with atezolizumab, pegylated liposomal doxorubicin and cyclophosphamide

2. Assessment of progression-free survival

Secondary objectives:

3. Assessment of clinical response: Objective tumor response rate (ORR), duration of response (DR), durable tumor response rate (DRR; $>6$ months), clinical benefit rate (CBR), overall survival (OS)

4. Assessment of changes in the immunological milieu in tumour and peripheral blood induced by the study therapy 
5. Assessment of PD-L1 expression, mutation load and immune gene expression as biomarkers for clinical response

6. Assessment of patient reported outcomes, as measured by the Chalder Fatigue Questionnaire (FQ), an 11-point Numerical Rating Scale (NRS) for pain intensity and EORTC QLQ-C15-PAL

7. Assessment of immunological response

8. Identification of biomarkers for clinical response, toxicity and immune response

9. Characterization of tumor evolution induced by the study therapy

10. Characterization of changes in microbiota induced by the study therapy

\section{Study design}

This is a randomized, double-blind, placebo-controlled exploratory phase II study evaluating the safety and efficacy of atezolizumab when combined with immunogenic chemotherapy in subjects with metastatic triple-negative breast cancer. Atezolizumab, pegylated liposomal doxorubicin and cyclophosphamide are the Investigational Medicinal Products (IMPs).

The patients $(n=75)$ will be randomized 2:3 into two arms (A:B):

- Arm A: Chemo (pegylated liposomal doxorubicin + cyclophosphamide) + placebo

- Arm B: Chemo (pegylated liposomal doxorubicin + cyclophosphamide) + atezolizumab

Upon radiographic disease progression per iRECIST, the patients must stop study treatment.

Patients that receive $\leq 3$ doses of atezolizumab/placebo or $\leq 2$ doses with PLD will not be considered evaluable per protocol, but will be included in the intention to treat (ITT) analysis.

To include a sufficient number of patients (75) in the per protocol (PP) analysis, the same number of patients will be added to study. The new subjects will be randomized according to the initial 2:3 ratio.

\section{Study treatment}

- Atezolizumab (or placebo) will be administered intravenously $840 \mathrm{mg}$ every 2 nd week until disease progression or for a maximum of 24 months

- Chemotherapy will be administered as follows:

- Pegylated liposomal doxorubicin $20 \mathrm{mg} / \mathrm{m}^{2}$ i.v. every 2 nd week. An upper limit of $44 \mathrm{mg}$ per dose will be applied to patients with a body surface area $>2.2 \mathrm{~m}^{2}$

- Cyclophosphamide tablets $50 \mathrm{mg}$ per day, daily as continuous treatment for the first 2 weeks in each 4 week period (i.e. every second 2 week cycle)

- No upper limit for the number of cycles of pegylated liposomal doxorubicin/cyclophosphamide. Heart function will be monitored with regard to pegylated liposomal doxorubicin

\section{Rationale for chemotherapy regime}

The chemotherapy regime used in the study is regarded as appropriate therapy for this patient group, without atezolizumab. The regime is expected to be well tolerated and applicable to most metastatic TNBC patients with ECOG 0-1, while also being sufficiently potent to suit those with an excellent performance status.

The chemotherapy regime and dosing schedule have been tailored to aid the effect of atezolizumab, which depends on immune effector cells for its activity. First, the chosen chemotherapeutic agents (anthracyclines and cyclophosphamide) are known to induce immunogenic cell death. Secondly, we apply the outlined dosage regime, rather than a high dose regime administered every $3 \mathrm{rd} / 4$ th week, in order to maintain the leukocyte counts and the ability of the effector immune cells to respond. Anthracyclines are routinely administered at intervals ranging from one to four weeks in metastatic TNBC patients. Tregs and MDSCs represent important mediators of tumor tolerance and may oppose the effect of atezolizumab. The metronomic cyclophosphamide dosage chosen in the present study has been widely used to counter Tregs [12] and MDSCs and is also considered safe, as it has been combined with multiple other chemotherapeutic agents without causing important toxicity [15]. We include 14 day intervals without cyclophosphamide to allow for unsuppressed $\mathrm{T}$ cell proliferation and activity, which may be important for the atezolizumab effect.

We will use pegylated liposomal doxorubicin to minimize the adverse effects of anthracyclines on the heart and allow for continued treatment beyond the otherwise mandatory anthracycline limits. The possibility of longterm treatment is important in order to appropriately test checkpoint inhibitors like atezolizumab, as these drugs are known to induce durable responses in other patient groups. Pegylated liposomal doxorubicin is also administered without the need for corticosteroids, which is desirable for immunotherapy.

The standard pegylated liposomal doxorubicin dosage for breast cancer is $40-50 \mathrm{mg} / \mathrm{m}^{2}$ every 4 th week. In Norway, the most widely used dose is $40 \mathrm{mg} / \mathrm{m}^{2}$. The 
dose chosen in our study is expected to be well tolerated, as the $40 \mathrm{mg} / \mathrm{m}^{2}$ is divided into two doses of $20 \mathrm{mg} / \mathrm{m}^{2}$ given every 2 nd week. Some studies in breast cancer have used pegylated liposomal doxorubicin at $15-30 \mathrm{mg} / \mathrm{m}^{2}$ every 2nd week [15-19], or in combination with cyclophosphamide $\left(500 \mathrm{mg} / \mathrm{m}^{2}\right)$, and 5 -fluorouracil $(500 \mathrm{mg} /$ $\mathrm{m}^{2}$ ) every 3rd week [20]. A dose of $20 \mathrm{mg} / \mathrm{m}^{2}$ has been well tolerated in combination with cyclophosphamide (50 mg/day) even in fragile, older patients [19] and is also tolerated by HIV positive patients with Kaposi sarcoma.

\section{Sample collection/biobanking}

Samples are collected before, during and after therapy (time of progression/treatment discontinuation). Some of the biobanking procedures are not performed at all study centers. The following samples are collected: Biopsies, peripheral blood mononuclear cells (PBMC), plasma, serum, urine, feces and circulating tumor cells.

\section{Selected inclusion criteria}

1. Metastatic or incurable locally advanced, histologically documented TNBC (absence of HER2, ER, and $\mathrm{PR}$ expression). ER and PR negativity are defined as $<1 \%$ and $<10 \%$, respectively, of cells expressing hormonal receptors by IHC analysis

2. Adequate core or excisional study biopsy of a tumor lesion.

3. Measurable metastatic disease according to iRECIST

4. Eastern Cooperative Oncology Group (ECOG) performance status of 0 or 1

5. Signed Informed Consent Form

6. Women or men aged $\geq 18$ years

7. A minimum of 12 months from adjuvant/neoadjuvant chemotherapy with anthracyclines to relapse of disease.

8. A maximum of one previous line of chemotherapy in the metastatic setting

9. Adequate organ function as defined in the protocol

\section{Selected exclusion criteria}

1. Malignancies other than breast cancer within 5 years prior to randomization, with the exception of those with a negligible risk of metastasis or death and treated with expected curative outcome

2. Spinal cord compression not definitively treated with surgery and/or radiation, or previously diagnosed and treated spinal cord compression without evidence that disease has been clinically stable for $>2$ weeks prior to randomization
3. Known CNS disease, except for treated asymptomatic CNS metastases, provided all of the following criteria are met:

a. Measurable disease outside the CNS

b. No metastases to mesencephalon, pons, medulla oblongata, or spinal cord

c. No evidence of progression after completion of CNS-directed therapy

d. No ongoing requirement for dexamethasone as therapy for CNS disease

e. No radiation of brain lesions within 14 days prior to randomization

f. No leptomeningeal disease

g. Patients with new asymptomatic CNS metastases detected at the screening scan must receive radiation therapy and/or surgery for CNS metastases. Following treatment, these patients may be eligible without the need for an additional brain scan prior to enrolment, if all other criteria are met.

4. Uncontrolled pleural effusion, pericardial effusion, or ascites.

5. Pregnant or breastfeeding

6. Received treatment with immune checkpoint modulators

7. Received treatment with systemic corticosteroids or other systemic immunosuppressive medications within 2 weeks prior to randomization, or anticipated requirement for systemic immunosuppressive medications during the trial

a. Patients who have received acute, low-dose, systemic immunosuppressant medications (e.g., a one-time dose of dexamethasone for nausea) may be enrolled in the study

b. Patients with a history of allergic reaction to IV contrast requiring steroid pre-treatment should have baseline and subsequent tumor assessments performed using MRI

c. The use of inhaled corticosteroids for chronic obstructive pulmonary disease, mineralocorticoids (e.g., fludrocortisone) for patients with orthostatic hypotension, and low-dose supplemental corticosteroids for adrenocortical insufficiency are allowed

8. Received anti-cancer therapy (medical agents or radiation) within 2 weeks prior to study Cycle 1, Day 1 . Palliative radiotherapy for bone lesions is allowed up to 2 weeks before start of therapy. 


\section{Outcome measures}

\section{Safety outcome measures}

The safety outcome measures will be evaluated in the ITT population, as follows:

- Incidence, nature, and severity of adverse events graded according to NCI CTCAE v4.0

- Changes in vital signs, physical findings, and clinical laboratory results

\section{Efficacy outcome measures}

The PFS is defined as the time from randomization to the time of radiographic progression (as assessed by iRECIST) or death from any cause during the study. Data for patients with a PFS event who missed two or more assessments scheduled immediately prior to the date of the PFS event will be censored at the last tumor assessment prior to the missed visits. If no tumor assessment was performed after randomization, data will be censored at the date of randomization +1 day. Clinical deterioration without objective radiological evidence will not be considered as documented disease progression. The primary efficacy outcome measure (PFS) is to be assessed in patients evaluable per protocol (PP).

The secondary efficacy outcome measures will be assessed in the PP population, ITT population and in the PD-L1-positive subpopulation.

\section{Statistics}

A descriptive analysis of demographics, medical history, and clinical data will be performed.

The ITT population is defined as a full analysis set (FAS). The FAS is defined as all patients that have started therapy with at least one of the IMPs, and where data on the relevant endpoint is obtained. The safety will be evaluated in the ITT population.

The primary efficacy analyses will be performed on the patients that have completed at least 4 doses of atezolizumab/placebo and 3 doses of PLD (PP population). Secondary efficacy analyses will be performed on the PD-L1 positive subpopulation and on the ITT (FAS) population.

The primary efficacy analysis will be a descriptive analysis of progression free survival (PFS) in the atezolizumab arm (B), compared to the control arm (A). The HR for disease progression or death ( $\operatorname{arm} B$ versus arm $A$ ) will be estimated using a Cox proportional hazards model. The confidence interval for the HR will be provided. KaplanMeier methodology will be used to estimate the median PFS for each treatment arm, and Kaplan-Meier curves will be produced.

Overall survival (OS) will be calculated from the time of randomization until death. Patients alive at the time of data analysis will be treated as censored. The HR for OS (arm B versus arm A) will be estimated using a Cox proportional hazards model. The CI for the HR will be provided. Kaplan-Meier methodology will be used to estimate the median OS for each treatment arm, and Kaplan-Meier curves will be produced.

Exploratory analyses will be carried out to evaluate the data of the immunological and molecular analyses (e.g. biomarker studies) carried out. The statistical analyses will be dependent on the biological factors investigated and the analysis methodology used, and will be defined separately for each molecular study.

We expect to reach the data-driven time point for PFSanalysis (90\% PFS events in the PP population) about 6 months after inclusion of the last patient. If this is not met within 12 months after inclusion of the last patient, the PFS-analysis will be performed at this time point. The primary data analysis will be performed on the PP population. Further, the following factors will be studied:

- Tumor PD-L1 status

- Disease free interval: Less than 24 months versus $>24$ months between end of adjuvant chemotherapy or surgery, whichever was last, and relapse. (Most TNBC patients receive adjuvant chemo for 6 months before/after radical surgery; a short disease-free interval suggests aggressive disease.)

- Prior chemotherapy against metastatic disease (no previous chemo versus previous chemo). Chemotherapy given in the neoadjuvant/adjuvant setting is not to be considered in this analysis

- Site(s) of metastases

- Molecular breast cancer profile and immune gene profile

\section{Discussion}

\section{Study organization and timeline}

Oslo University Hospital, Oslo, Norway, is the study sponsor. We have established 3 study centers in Norway (Oslo University Hospital, Stavanger University Hospital, St Olav University Hospital Trondheim) and two in Denmark Denmark (Rigshospitalet Copenhagen and Vejle University Hospital). The study opened in 2017, expanded to more sites in 2018 and has included 48 patients as of March 2020. We estimate a need to recruit 85 patients, to obtain the required 75 evaluable patients per protocol.

\section{Comments on study design and objectives}

In the ALICE study, and a parallel trial in hormone receptor positive breast cancer (ICON; NCT03409198), we have chosen a randomized phase IIb design, with a 
limited number of patients. Historic controls are heterogeneous and of limited value. The key issue is that neither the effect nor the toxicity of adding a PD-L1 inhibitor to chemotherapy can be properly assessed in a one-armed study. On the other hand, a full-scale phase III trial, powered to show clinical efficacy with a $p<0.05$, is not warranted, too resource demanding and ethically difficult, until a certain level of clinical data have been generated in TNBC.

The primary objective of the ALICE study is to assess the toxicity of atezolizumab as an add-on to chemotherapy, and provide leads on potential clinical efficacy against TNBC. Accordingly, the study was not powered to demonstrate a statistically significant $(p<0.05)$ clinical effect. If the study suggests acceptable toxicity and potential clinical benefit, a larger randomized study will be warranted.

Contrary to many phase II trials, the study includes a comprehensive assessment of quality of life (QoL). It is well known that the doctor's perception and grading of side effects does not always correspond to the patient's well-being. Further, the clinical efficacy in the palliative setting is certainly dependent on the effect on symptoms, not only survival time or objective tumour response. We aim to capture these aspects by use of a vailidated general form (EORTC QLQ-C15-PAL), tailored for metastatic patients, as well as specific read-outs for two key symptoms-fatigue and pain. The latter two symptoms were selected because they are considered to be important in this patient group.

Another key objective in ALICE is to assess the changes in the immunological milieu after study therapy. We will evaluate if immune activation is induced in the tumour by analysing repeated biopsies. Further, we will assess the effect of the study therapy at systemic level, by analyses of peripheral mononuclear blood cell, plasma and serum. Each of the study arms will be analysed separately, and compared.

The biomarker analyses represent another important objective in the study. These analyses, and other translational projects included in ALICE, may inform the selection of patient subgroups for later studies, identify strategies for improved therapy and allow for more personalized approaches. We aim to investigate mechanisms of effect and resistance, and assess the dynamic changes and tumor-immune co-evolution induced by the therapy. To this aim, consecutive biopsies, PBMCs and other samples are obtained.

Among the key challenges facing the field, is the question of which chemotherapy should be added to PD-L1/ PD-1 blockers, and how the chemotherapy dosage should be tailored. In theALICE trial, we have in part based the choice of chemotherapy on current knowledge about immunogenic cell death and other effects of chemotherapy. Importantly, most of this knowledge is derived from preclinical models. There is sparse evidence from patients regarding the hypothesized beneficial effects of anthracyclines (immunogenic cell death) and low-dose cyclophosphamide (counter Tregs/MDSCs). A critical evaluation of these hypotheses, as included in the ALICE study, is required to inform the choice and dosing of chemotherapy for combination with checkpoint inhibitors in TNBC. The findings from ALICE may also be relevant to other cancer forms.

\section{Abbreviations}

CBR: Clinical benefit rate; Cl: Checkpoint inhibitor; CNS: Central nervous system; CR: Complete response; CTC: Circulating tumor cells; CTCAE: Common terminology criteria for adverse event; DOR: Duration of objective response; DR: Duration of response; DRR: Durable tumor response rate; ECOG: Eastern cooperative oncology group; EMA: European medicines agency; ER: Estrogen receptor; FAS: Full analysis set; HER2: Human epidermal growth factor receptor 2; HR: Hazard ratio; ICH: International conference on harmonization; IHC: Immunohistochemistry; IMP: Investigational medicinal product; ISH: In situ hybridization; ITT: Intention to treat; IV: Intravenous; MDSC: Myeloid-derived suppressor cells; ORR: Overall response rate; OS: Overall survival; PBMC: Peripheral blood mononuclear cell; PD: Progressive disease; PD-1: Programmed death 1; PD-L1: Programmed death ligand-1; PFS: Progression-free survival; PLD: Pegylated liposomal doxorubicin; PP: Per protocol; PR: Progesterone receptor; QLQ: Quality of life questionnaire; SD: Stable disease; TNBC: Triplenegative breast cancer; Tregs: T regulatory cells.

\section{Acknowledgements}

The authors thank doctors at the Breast Cancer Unit, study nurses and project managers at the Clinical Research Unit and pathologists at Department of Pathology for important input during the preparation of the trial. Special thanks to Dr. Elin Borgen, Dr. Hege Russnes, Ismail Abdi and Professor Erik Wist for valuable advice. We also thank Roche, The Norwegian Breast Cancer Society, the Norwegian Cancer Society and the South-Eastern Norway Regional Health Authority for supporting the trial with funding and free study drug.

\section{Authors' contributions}

JAK: Principal investigator; concept, preparation of trial, writing of protocol and manuscript. AR: Study doctor; contribution to protocol revisions and manuscript. RSF: Study statistician; contribution to protocol and manuscript. BN: Co-investigator; contribution to protocol, trial preparations and manuscript. All authors read and approved the final manuscript.

\section{Funding}

Roche supplies atezolizumab free of charge and provides a funding contribution. The study is supported by a Pink Ribbon grant from the Norwegian Cancer Society and Breast Cancer Society, and by a grant from the South-Eastern Norway Regional Health Authority. Nanostring and Menarini provide assays/ kits free of charge for translational research. The expenses for chemotherapy, routine blood samples and radiology are covered by the national health care systems. Funding was provided by Kreftforeningen (Grant No. 182632-2016), Helse Sør-Øst RHF (Grant Nos. 2017100, 2017122), Roche.

\section{Availability of data and materials}

Not applicable.

\section{Ethics approval and consent to participate}

The ALICE trial has been approved by the Norwegian and Danish Medical Agencies and Ethics Committees. The study is performed in compliance with the World Medical Association Declaration of Helsinki and ICH E6 for Good Clinical Practice. Signed informed consent is obtained from all patients. 


\section{Consent for publication}

All authors have consented to the publication. Approval has also been obtained from Roche.

\section{Competing interests}

The authors declare that they have no competing interests.

\section{Author details}

1 Department of Clinical Cancer Research, Oslo University Hospital, Oslo, Norway. ${ }^{2}$ Department of Cancer Immunology, Oslo University Hospital, Oslo, Norway. ${ }^{3}$ Department of Oncology, Oslo University Hospital, Oslo, Norway.

${ }^{4}$ Oslo Centre for Biostatistics and Epidemiology, Oslo University Hospital, Oslo, Norway. ${ }^{5}$ Institute of Clinical Medicine, University of Oslo, Oslo, Norway.

Received: 27 April 2020 Accepted: 17 June 2020

Published online: 23 June 2020

\section{References}

1. Bianchini G, et al. Triple-negative breast cancer: challenges and opportunities of a heterogeneous disease. Nat Rev Clin Oncol. 2016;13(11):674-90.

2. Larkin J, et al. Combined nivolumab and ipilimumab or monotherapy in untreated melanoma. N Engl J Med. 2015;373(1):23-34.

3. Rizvi NA, et al. Cancer immunology. Mutational landscape determines sensitivity to PD-1 blockade in non-small cell lung cancer. Science. 2015;348(6230):124-8.

4. Ferris RL, et al. Nivolumab for recurrent squamous-cell carcinoma of the head and neck. N Engl J Med. 2016;375(19):1856-67.

5. Schmid $\mathrm{P}$, et al. Atezolizumab and nab-paclitaxel in advanced triplenegative breast cancer. N Engl J Med. 2018;379(22):2108-21.

6. Schmid $\mathrm{P}$, et al. Atezolizumab plus nab-paclitaxel as first-line treatment for unresectable, locally advanced or metastatic triple-negative breast cancer (IMpassion130): updated efficacy results from a randomised, doubleblind, placebo-controlled, phase 3 trial. Lancet Oncol. 2020;21(1):44-59.

7. Pfirschke $C$, et al. Immunogenic chemotherapy sensitizes tumors to checkpoint blockade therapy. Immunity. 2016:44(2):343-54.

8. Kroemer $\mathrm{G}$, et al. Natural and therapy-induced immunosurveillance in breast cancer. Nat Med. 2015:21(10):1128-38.
9. Bezu L, et al. Combinatorial strategies for the induction of immunogenic cell death. Front Immunol. 2015;6:187.

10. Sistigu A, et al. Cancer cell-autonomous contribution of type I interferon signaling to the efficacy of chemotherapy. Nat Med. 2014;20(11):1301-9.

11. Apetoh $\mathrm{L}$, et al. Toll-like receptor 4-dependent contribution of the immune system to anticancer chemotherapy and radiotherapy. Nat Med. 2007:13(9):1050-9.

12. Ghiringhelli F, et al. Metronomic cyclophosphamide regimen selectively depletes CD4+CD25+ regulatory T cells and restores T and NK effector functions in end stage cancer patients. Cancer Immunol Immunother. 2007;56(5):641-8.

13. Voorwerk $L$, et al. Immune induction strategies in metastatic triple-negative breast cancer to enhance the sensitivity to PD-1 blockade: the TONIC trial. Nat Med. 2019;25(6):920-8.

14. Schmid $\mathrm{P}$, et al. Pembrolizumab for early triple-negative breast cancer. $\mathrm{N}$ Engl J Med. 2020;382(9):810-21.

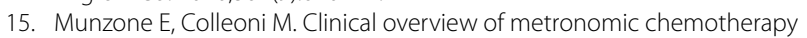
in breast cancer. Nat Rev Clin Oncol. 2015;12(11):631-44.

16. Zhao M, et al. Use of liposomal doxorubicin for adjuvant chemotherapy of breast cancer in clinical practice. J Zhejiang Univ Sci B. 2017;18(1):15-26

17. Jehn CF, et al. Biweekly pegylated liposomal doxorubicin (Caelyx) in heavily pretreated metastatic breast cancer: a phase 2 study. Clin Breast Cancer. 2016;16(6):514-9.

18. Rossi D, et al. Neoadjuvant chemotherapy with low dose of pegylated liposomal doxorubicin plus weekly paclitaxel in operable and locally advanced breast cancer. Anticancer Drugs. 2008;19(7):733-7.

19. Dellapasqua $S$, et al. Pegylated liposomal doxorubicin in combination with low-dose metronomic cyclophosphamide as preoperative treatment for patients with locally advanced breast cancer. Breast. 2011:20(4):319-23.

20. Rau KM, et al. Pegylated liposomal doxorubicin (Lipo-Dox(R)) combined with cyclophosphamide and 5-fluorouracil is effective and safe as salvage chemotherapy in taxane-treated metastatic breast cancer: an open-label, multi-center, non-comparative phase II study. BMC Cancer. 2015:15:423.

\section{Publisher's Note}

Springer Nature remains neutral with regard to jurisdictional claims in published maps and institutional affiliations.
Ready to submit your research? Choose BMC and benefit from:

- fast, convenient online submission

- thorough peer review by experienced researchers in your field

- rapid publication on acceptance

- support for research data, including large and complex data types

- gold Open Access which fosters wider collaboration and increased citations

- maximum visibility for your research: over $100 \mathrm{M}$ website views per year

At $\mathrm{BMC}$, research is always in progress.

Learn more biomedcentral.com/submissions 